\title{
Targeting Follistatin like 1 ameliorates liver fibrosis induced by carbon tetrachloride through TGF- $\beta 1-$ miR29a in mice
}

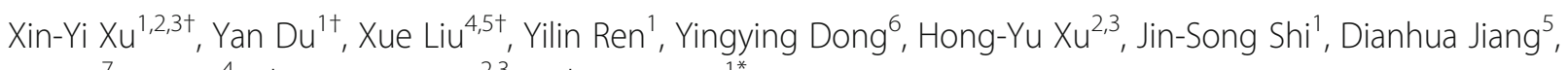
$\mathrm{Xin} \mathrm{Xu}^{7}$, Lian $\mathrm{Li}^{4}$, Zheng-Hong $\mathrm{Xu}^{2,3}$ and Yan Geng ${ }^{1 *}$

\begin{abstract}
Background: Hepatic fibrosis is a pathological response of the liver to a variety of chronic stimuli. Hepatic stellate cells (HSCs) are the major source of myofibroblasts in the liver. Follistatin like 1 (Fstl1) is a secreted glycoprotein induced by transforming growth factor- $\beta 1$ (TGF- $\beta 1$ ). However, the precise functions and regulation mechanisms of Fstl1 in liver fibrogenesis remains unclear.

Methods: Hepatic stellate cell (HSC) line LX-2 stimulated by TGF- $\beta 1$, primary culture of mouse HSCs and a model of liver fibrosis induced by $\mathrm{CCl} 4$ in mice was used to assess the effect of Fstl1 in vitro and in vivo.

Results: Here, we found that Fstl1 was significantly up regulated in human and mouse fibrotic livers, as well as activated HSCs. Haplodeficiency of Fst/1 or blockage of Fstl1 with a neutralizing antibody $22 \mathrm{~B} 6$ attenuated $\mathrm{CCl}_{4}$-induced liver fibrosis in vivo. Fstl1 modulates TGF- $\beta 1$ classic Samd2 and non-classic JNK signaling pathways. Knockdown of Fstl1 in HSCs significantly ameliorated cell activation, cell migration, chemokines C-C Motif Chemokine Ligand 2 (CCL2) and C-X-C Motif Chemokine Ligand 8 (CXCL8) secretion and extracellular matrix (ECM) production, and also modulated microRNA-29a (miR29a) expression. Furthermore, we identified that Fstl1 was a target gene of miR29a. And TGF- $\beta 1$ induction of Fstl1 expression was partially through down regulation of miR29a in HSCs.
\end{abstract}

Conclusions: Our data suggests TGF- $\beta 1$-miR29a-Fstl1 regulatory circuit plays a key role in regulation the HSC activation and ECM production, and targeting Fst1 1 may be a strategy for the treatment of liver fibrosis.

Keywords: Hepatic fibrosis, Cell differentiation, Transforming growth factor- $\beta$ (TGF- $\beta$ ) signaling, Follistatin like 1 (Fst11), microRNA

\section{Background}

Liver fibrosis is a scarring process that occurs in most chronic liver diseases, including nonalcoholic fatty liver disease (NAFLD), alcoholic liver disease, and hepatitis B/C virus infection. Hepatic stellate cells (HSCs), liver specific mesenchymal cells, are the primary cell type responsible for the development of liver fibrosis. HSCs contain

\footnotetext{
* Correspondence: gengyan@jiangnan.edu.cn

${ }^{\dagger}$ Xin-Yi Xu, Yan Du and Xue Liu contributed equally to this work.

${ }^{1}$ School of Pharmaceutical Sciences, Jiangnan University, Wuxi 214122, China Full list of author information is available at the end of the article
}

numerous lipid droplets in normal liver. During chronic liver injuries, HSCs are activated by cytokines and chemokines from damaged hepatocytes and immune cells, then transdifferentiate to myofibroblasts that produce massive ECM and fibrogenic cytokines [1, 2]. The activated HSCs, in turn, release more cytokines and chemokines, leading to enhanced inflammatory responses in injury area [3]. Among many cytokines mediating the fibrotic cascades, transforming growth factor- $\beta$ (TGF- $\beta$ ) is a central profibrotic growth factor [4]. TGF- $\beta$ plays a key role in HSCs activation, migration and transdifferentiation into

C C The Author(s). 2020 Open Access This article is licensed under a Creative Commons Attribution 4.0 International License, which permits use, sharing, adaptation, distribution and reproduction in any medium or format, as long as you give appropriate credit to the original author(s) and the source, provide a link to the Creative Commons licence, and indicate if changes were made. The images or other third party material in this article are included in the article's Creative Commons licence, unless indicated otherwise in a credit line to the material. If material is not included in the article's Creative Commons licence and your intended use is not permitted by statutory regulation or exceeds the permitted use, you will need to obtain permission directly from the copyright holder. To view a copy of this licence, visit http://creativecommons.org/licenses/by/4.0/ The Creative Commons Public Domain Dedication waiver (http://creativecommons.org/publicdomain/zero/1.0/) applies to the data made available in this article, unless otherwise stated in a credit line to the data. 
myofibroblasts, as well as simulating the synthesis of ECM $[5,6]$. Hepatic specific overexpression of mature TGF- $\beta 1$ leads to liver fibrosis in mice [7]. Blocking TGF- $\beta 1$ signaling pathway by TGF- $\beta 1$ antibodies or antisense oligonucleotides, and soluble TRRII attenuated liver fibrosis in experimental models [8].

MicroRNAs (miRNAs) are endogenous 20-22 nucleotides RNAs that control translation and transcription of many genes. MicroRNA-29 families $(m i R-29 a / b / c)$ [9] are known to be the downstream target of TGF- $\beta$ and play fundamental roles in liver [10, 11], lung [12, 13], and cardiac fibrosis [14]. Members of miR-29 family were down regulated in HSCs activation in vitro and in fibrotic livers in human and mice [10,11, 15]. Moreover, patients with liver fibrosis showed significantly lower levels of circulating miR-29a, when compared with healthy controls [10]. Ectopic expression of miR-29b in the liver of mice attenuated $\mathrm{CCl}_{4}$ induced liver fibrosis [11]. However, mechanism of action of $m i R-29 a$ in liver fibrosis remains largely unclear.

Follistatin-like 1 (Fstl1) is a secreted glycoprotein belonging to the Follistatin (Fst) family and secreted protein acidic rich in cysteines (SPARC) family [16], which can be induced by TGF- $\beta$ [17]. Although Fst expression was unchanged in activated HSCs, Fst treatment ameliorated early liver fibrosis in experimentally induced liver fibrosis in rats by blocking Activin bioactivity [18]. SPARC expression in hepatic tissue was significantly increased during the development of liver fibrosis, and targeting SPARC through an adenovirus carrying antisense SPARC suppressed HSCs activation in thioacetamide induced liver fibrosis in rats [19]. As the smallest member in the Fst-SPARC family, the role of Fstl1 in liver fibrosis and its therapeutic potential has not been fully investigated.

Homozygous $\mathrm{Fstl1}^{-1-}$ mice die of respiratory failure shortly after birth [20], so Fstl1 ${ }^{+-}$or conditional knockout mice have been used to study the lung and kidney fibrosis [21, 22]. The results showed that haplodeficiency of Fstl1 or blockage of Fstl1 with a neutralizing antibody attenuated bleomycin induced lung fibrosis in mice [21]. Cardiac-specific Fstl1deficient mice promoted tubulointerstitial fibrosis after subtotal renal ablation compared with wild-type mice [22]. In addition, application of the human FSTL1 protein via an epicardial patch stimulates pre-existing cardiomyocytes proliferation, improves cardiac function and attenuated fibrosis in animal models of myocardial infarction [23]. Northern blot analysis of murine tissues showed there was barely any Fstl1 transcript in the liver [24]. Recently, Fstl1 was identified as a fibrosis modifier by in vivo siRNA silencing screen [25]. Knockdown Fstl1 suppressed HSCs activation [26]. These data indicate that the role of Fstl1 in tissue fibrosis is controversial.
RNA deep sequencing and function assays revealed that FSTL1 may be an endogenous target of miR-29a in human myotubes [27]. MiR-29a can promote the neurite outgrowth by targeting extracellular matrix related genes including Fstl1 [28]. In this study, we aim to analyze the role of Fstl1 in liver fibrosis by using TGF- $\beta 1$ activated HSCs in vitro and a mouse model of $\mathrm{CCl}_{4}$-induced liver fibrosis. We found that Fstll is evolved in the pathogenesis of liver fibrosis through a TGF- $\beta 1$-miR29a-Fstl1 regulatory circuit and can serve as a therapeutic target for the treatment of liver fibrosis.

\section{Methods}

\section{Chemicals and reagents}

$\mathrm{CCl}_{4}$ and Olive Oil were from Sigma-Aldrich (St Louis, MO, USA). Fstl1 neutralizing antibody was generated as described previously [21]. Fstl1 siRNA and scramble RNA were purchased from Genechem Company (Shanghai, China). The mimics and inhibitor of miR29a were purchased from Ruibo Company (Guangdong, China). The $\alpha$ SMA, GAPDH antibodies were purchased from Santa Cruz Biotechnology (USA). The Fstl1 antibodies were purchased from Santa Cruz Biotechnology (USA) or R\&D systems (USA). Smad2, p-Smad2, p-JNK and JNK were purchased from Cell Signaling Technology (USA).

\section{Subjects}

The study was approved by the Institutional Review Board of Wuxi No.2 People's Hospital (No. 20170608) and were in accordance with the principles of the Declaration of Helsinki as revised in 2000 . The study includes 27 healthy controls and 19 patients (Table S1). All participants signed a written consent form before entering the study. All patients included in this study were diagnosed according to the respective diagnostic criteria. The healthy volunteers were recruited from the medical examination center of Jiangnan University that had normal aminotransferase activities, no history of liver disease or alcohol abuse and tested negative for HBV, HCV and HIV infections. Paraffin liver sections (LV1201) were from Alenabio.com (Xi'an, China). All human tissues are collected under IRB and HIPPA approved protocols, and approved for commercial product development.

\section{Enzyme-linked immunosorbent assay (ELISA)}

The amount of FSTL1 (Cloud-Clone, USA) in serum and C-C Motif Chemokine Ligand 2 (CCL2) (Sino Biological, Beijing) or C-X-C Motif Chemokine Ligand 8 (CXCL8) (Invitrogen, USA) released from LX-2 cells into the culture medium was determined using commercially available ELISA kits according to the manufacturer's instructions. Recombinant standards of FSTL1, CCL2 or CXCL8 provided in the kit and the serum or isolated culture medium were added to a plate pre- 
coated with a monoclonal antibody against the chemokine. After incubation for $1 \mathrm{~h}$, the plate was washed and incubated with an enzyme-linked polyclonal antibody specific for FSTL1, CCL2 or CXCL8. After several washes, the substrate solution was added, and the color intensity was measured. A standard curve was used for determination of the amount of FSTL1, CCL2 or CXCL8 present in the samples.

\section{Animal model of liver fibrosis and treatment}

The Animal Research Committee of Jiangnan University and Nankai University approved all animal experiments. Male C57BL/6 or BABL/c mice at 8 weeks were purchased from Shanghai Slac Laboratory Animal CO.LTD (China). Fstl1 ${ }^{+-}$mice were described previously [20] and backcrossed to C57BL/6 background for more than ten generations. The mice were allowed free access to tap water and a chow diet (M01-F25-20150922034, Shanghai SLAC Laboratory Animal Co., Shanghai, China). Liver fibrosis was induced by intraperitoneal (i.p.) injection of $0.5 \mathrm{ml} / \mathrm{kg} \mathrm{CCl}$ (25\% solution in olive oil) twice per week [29]. At designated time points after $\mathrm{CCl}_{4}$ or olive oil injection, mice were euthanized with phenobarbital sodium by i.p. injection, and livers were harvested for further analyses. Fstl1-neutralizing antibody (clone 22B6) or its control isotype antibody (IgG1) was intravenously injected $(25 \mu \mathrm{g} /$ mouse/each time $)$ along with $\mathrm{CCl}_{4}$ treatment. The mouse livers were harvested $28 \mathrm{~d}$ after $\mathrm{CCl}_{4}$ injury. Tissues were sectioned for Picro-sirius red (PSR) staining to assess the degree of fibrosis. Collagen contents in the liver were measured with a conventional hydroxyproline method [30].

\section{Primary hepatic stellate cells isolation, cell culture and drug treatment}

The isolation of HSCs from murine livers can be divided into three main sequential stages [31]. Briefly, the mouse livers were in situ perfused with pronase and collagenase. And then, the liver tissues were carefully removed, minced under sterile conditions and further digested with pronase/collagenase. At last, the HSCs were isolated with density gradient-based separation from other hepatic cell populations.

Human HSCs cell line LX-2 cells, rat HSCs cell line CFSC-8B and HSC-T6 were obtained from the cell bank of Xiangya Central Experiment Laboratory of Central South University (Changsha, China). Cells were cultured in DMEM or RPMI 1640 supplemented with $10 \%$ heatinactivated FBS (Gibco, USA) and antibiotics at $37^{\circ} \mathrm{C}$ in a humidified atmosphere of $5 \% \mathrm{CO}_{2}$. Cells were grown to $100 \%$ confluence and serum starved for $24 \mathrm{~h}$ before treatment. Cells were pretreated with $2 \mu \mathrm{g} / \mathrm{ml}$ antibody (22B6) or control IgG1 for $24 \mathrm{~h}$ and then treated with 5 ng/ml TGF- $\beta 1$. Fstll siRNA ( $40 \mathrm{nM})$, scramble control
RNA (40 nM), miR29a mimics $(100 \mathrm{nM})$ or inhibitors $(200 \mathrm{nM})$ were transiently transfected into LX-2 cells using Lipofectamine RNAi max (Invitrogen, CA, USA) for $48 \mathrm{~h}$.

\section{RNA isolation and qRT-PCR analysis}

Total RNA was extracted from mouse liver tissue or cells with Trizol reagent (Invitrogen, CA, USA). We performed RNA isolation and qRT-PCR analysis as previously described [23]. Gene expressions were measured relative to the endogenous reference gene Gapdh using the comparative CT method and the sequences of specific primer pairs for Fstll, $\alpha-S M A$, and Colla1 were described previously [21]. The expression level of mature miR-29a was quantified by TaqMan microRNA assays (Mm04238191_s1, Applied Biosystems, CA, USA).

\section{Western blot analysis}

Cells or liver tissues were washed with ice cold DPBS and re-suspended in RIPA buffer with protease inhibitor (Sigma-Aldrich, MO, USA). Protein was resolved by SDS-polyacrylamide gel electrophoresis and transferred to PVDF membranes. After blocking, they were probed with primary antibodies overnight at $4{ }^{\circ} \mathrm{C}$, then incubated with horseradish peroxidase-conjugated secondary antibody for $1 \mathrm{~h}$ at room temperature. The bands were visualized using ECL reagents (Thermofisher Scientific, USA). Band intensity on scanned films was quantified using Image lab software (Bio-Rad Laboratories, Inc. USA). The ratio of the relevant protein was subjected to internal control (GAPDH).

\section{Cell migration assays}

The cell migration assay was performed with Transwell chambers with $8-\mu \mathrm{m}$ pores (Corning, USA). LX-2 or primary mouse HSCs $\left(2.5 \times 10^{4}\right.$ cells per chamber $)$ in serum free medium were plated in the upper chambers in duplicate filters. DMEM containing 10\% heatinactivated FBS was added to the lower chamber as a chemoattractant. After $24 \mathrm{~h}$, non-migrating cells were removed from the upper surface, and filters were stained with crystal violet. Migrated cells were counted in five representative microscopic fields (100× magnification).

\section{Measurement of serum aminotransferase activities}

The activities of alanine aminotransferase (ALT) and aspartate aminotransferase (AST) in serum were estimated spectrophotometrically using commercial diagnostic kits (Jiancheng Institute of Biotechnology, Nanjing, China).

\section{Luciferase reporter assay}

The luciferase reporter assay was conducted using a Dual-Luciferase Reporter Assay System (Beyotime Biotechnology, China). PmiR-RB-Report ${ }^{\text {Tw }}$ vector is specially 
used to identify direct targets of microRNA. The Wildtype (WT) or mutant (Mut) 3 'UTR region of Fstl1 was cloned to the downstream of reporter Renilla luciferase gene (hRluc) by XhoI and NotI digestion. The primers for clone Fstl1-WT were 5'-GCG GCT CGA GGC AAT AAA GGA TAT GAA GGT GGC T-3' and 5' - AAT GCG GCC GCA TGA AGT GGT GGG ACT ACT GAA AA-3'. The primers for clone Fstl1-Mut were 5'TTA CCA AAC CAC GAT TTT CTC TGT AAA ACA CTT-3' and 5' - CAG AGA AAA TCG TGG TTT GGT AAA AAG TAT TTT-3'. LX-2 cells $\left(8.0 \times 10^{3} /\right.$ well $)$ were seeded into 96 -well plates for $24 \mathrm{~h}$ and then the cells were transiently co-transfected with pmiR-RBReport $^{\mathrm{tm}}$-Fstl1-WT/-Mut plasmids and miR29a mimics using Lipofectamine 3000 (Thermofisher, USA). Cells were lysed and assayed for Renilla luciferase activity $48 \mathrm{~h}$ after transfection. $100 \mu \mathrm{l}$ cell extracts were subjected to the Dual Luciferase Reporter Gene Assay Kit in Multiscan Spectrum. The firefly luciferase (hLuc) was used as internal control.

\section{Statistical analysis}

Data are expressed as means \pm SEM. Differences in measured variables between experimental and control groups were assessed by using Student's test. Differences in multiple groups were assessed by using one-way analysis of variance (ANOVA), and the Tukey test was used for determining the significance. Results were considered statistically significant at $P<0.05$. All analyses were conducted in Graphpad Prism software version 7.0.

\section{Results}

Over-expression of FSTL1 in Serum and Livers of Human Patients with Chronic liver diseases.

Serum concentrations of FSTL1 levels were determined for healthy controls (CTL) and patients with viral hepatitis B (HBV), cirrhosis (LC) and hepatocellular carcinoma (HCC). The result showed that FSTL1 levels were higher in patient groups than those in CTL (Fig. 1a, $\mathrm{P}<0.05$ and Table S1). FSTL1 immunostaining was weak in liver sections from CTL and was increased significantly (more than two-fold, $p<0.05$ ) in the cytoplasm of hepatocyte in liver sections from patients with HBV and LC which co-stained with $\alpha$-SMA (Fig. 1b). FSTL1 protein was also found aberrantly increased in HCC tissues compared to adjacent liver tissues [32]. Then we analyzed FSTL1 expression in a gene-profiling dataset of percutaneous liver biopsies from NAFLD patients [33] through The NCBI Gene Expression Omnibus (GEO accession:GSE31803). Clinicians rely upon the severity of liver fibrosis to segregate patients with NAFLD into subpopulation at low versus high-risk for eventual liverrelated morbidity and mortality. There was a significance increase in Fstl1 mRNA expression in liver tissues of high-risk (fibrosis stage 3-4) compared with low-risk (fibrosis stage 0-1) (Fig. 1c). These data indicate that FSTL1 may contribute to the progression of chronic liver diseases.

\section{Pathological expression of FSTL1 in fibrotic livers in $\mathrm{CCl}_{4}{ }^{-}$ injured mice}

Then we examined the expression of Fstl1 in a wellcharacterized murine model of $\mathrm{CCl}_{4}$ induced liver fibrosis. After 7, 14 and 28 days of repetitive $\mathrm{CCl}_{4}$ treatment, the gradually elevated expression of $\alpha-S M A$ and Col1 suggested the persistent existence of liver damage and scar formation. $\mathrm{CCl}_{4}$-induced injury stimulated Fstl1 expressions at mRNA and protein levels significantly after 28 days (Fig. 2a-d). We detected Fstl $1^{+}$cells co-stained with HSCs activation marker $\alpha$-SMA in mouse livers at 28 days after prolonged administration of $\mathrm{CCl}_{4}$ (Fig. 2e). Fstl1 was expressed higher levels in the HSCs than the other cell types in the liver, including hepatocyte, Kupffer cells, intrahepatic cholangiocytes and liver sinusoidal endothelial cells (LSECs) by Mass spectrometry-based proteomics (Fig. 2f) [34]. FSTL1 gene expression was significantly up-regulated (more than 5 fold) in human active cell line LX-2 compared with primary HSCs through DNA microarray analyses [35]. Furthermore, senescence of activated HSCs limits liver fibrosis [36]. Based on the GEO database (GEO accession: GDS3492), the gene expression of Fstl1 was significantly higher in growing activated HSCs than senescent HSCs stimulated by DNA damage drug etoposide (Figure S1). We also isolated the primary mouse HSCs (mHSCs) and confirmed the expression of $\alpha-S M A$ and Col1 were upregulated in in vitro culture. The expression level of Fstl1 was gradually increased during this activation progress (Fig. 2g-i). These data suggest that Fstl1 is a fibrosis related gene and may be critical for the activation of HSCs.

\section{Fst $11^{+/-}$mice have an attenuated fibrotic phenotype after liver injury}

To determine the role of Fstl1 in vivo, we subjected $\mathrm{Fstl}^{+/-}$and littermate wild type (WT) mice to the $\mathrm{CCl}_{4}$ induced liver fibrosis. $\mathrm{Fstl1}^{+--}$mice had significant less Fstl1 and $\alpha-S M A$ gene expression in fibrotic livers than that in the WT mice (Fig. 3a,b). The expression of Col1 was also reduced in $\mathrm{Fstll}^{+/-}$mice, whereas did not reach statistical significance (Fig. 3c). The protein expression level of Fstl1 and $\alpha$-SMA were downregulated in livers of $\mathrm{Fstl1}^{+/-}$mice compared with WT mice (Fig. 3d-f). $\mathrm{Fstl1}^{+/-}$mice also showed reduced degree of liver fibrosis, as determined by Sirius-red staining (Fig. 3g). These data indicate that Fstl1 is induced in response to liver injury and may promote live fibrosis in vivo. 

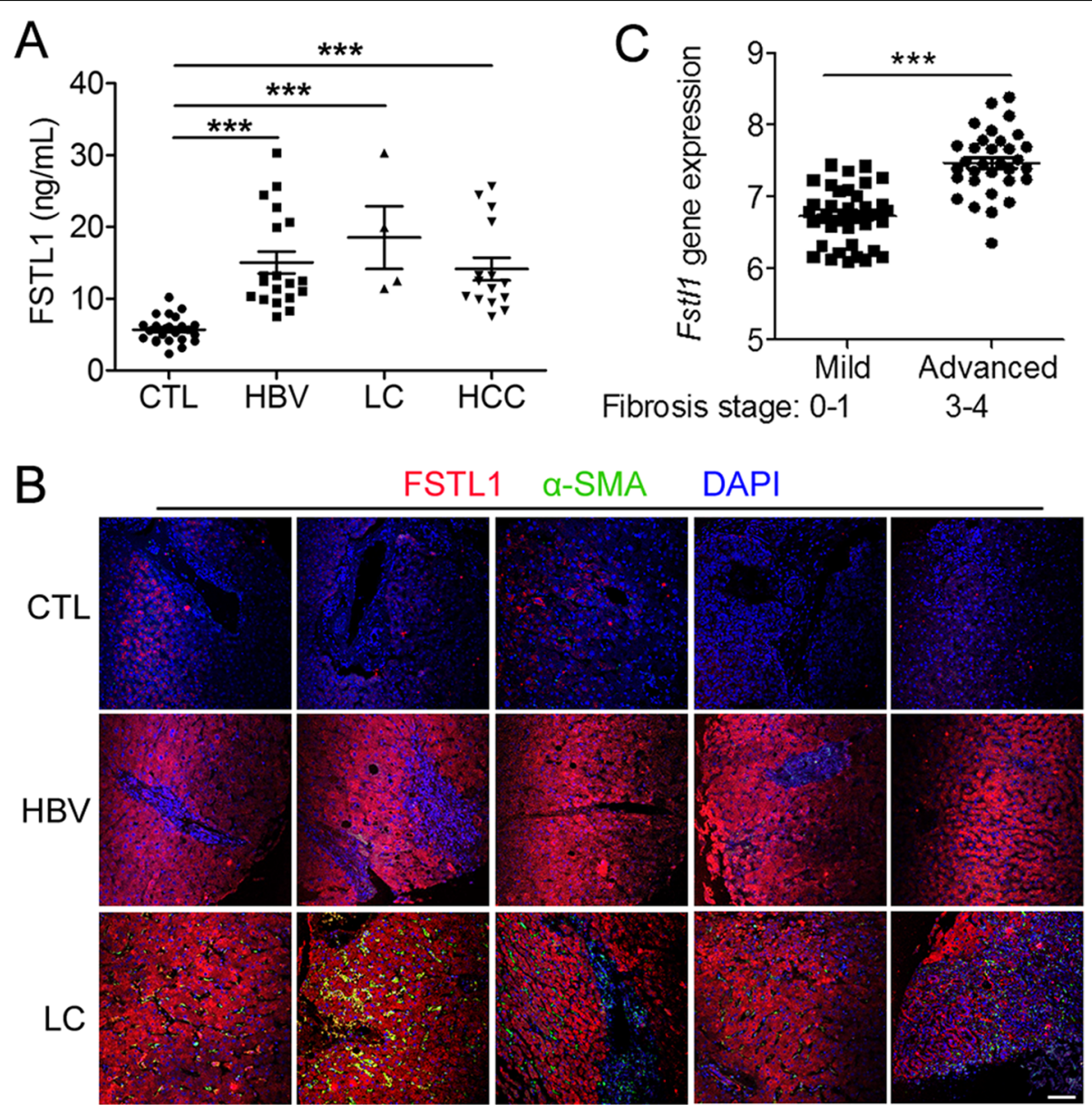

Fig. 1 FSTL1 expression in chronic liver diseases. a Serum concentrations of FSTL1 in healthy CTL $(n=27)$ and patients with HBV $(n=19)$, LC $(n=$ 4) and HCC $(n=15)$. b Paraffin liver sections from healthy CTL, and patients with HBV and LC were stained with FSTL1 (red), a-SMA (green) and DAPI ( $n=5$ per group). Scale bar: $100 \mu \mathrm{m}$. HBV, Viral Hepatitis B; LC, Cirrhosis; HCC, hepatocellular carcinoma. c FSTL1 expression in human liver tissues was examined in a published gene-profiling dataset (GEO: GSE31803). The degree of liver fibrosis is divided into mild ( $n=40)$ and severe forms $(n=32)$. Throughout, data represent mean \pm SEM. ${ }^{* *} P<0.001$

\section{Silencing Fstl1 inhibits HSCs activation in vitro}

To test whether TGF- $\beta 1$ might regulate the expression of Fstl1 in HSCs, human HSC cell line LX-2, rat HSC cell lines CFSC-8B and HSC-T6 were used. Gene and protein expression of Fstl1 were increased by TGF- $\beta 1$ in a time and dose dependent manner, correlating with increases in $\alpha$-SMA and Col1 expression in these cells (Figure S2-S3). FSTL1 siRNA was highly effective in decreasing Fstl1 gene and protein expression relative to a scramble siRNA control (Figure S4a-b). Decreased FSTL1 expression led to the inhibition of expression of $\alpha$-SMA and COL1 (Figure S4a-c). In addition, knockdown FSTL1 inhibited phosphorylation of JNK and TGF- $\beta 1$ induced phosphorylation of Smad2 (Figure S4bc). Chemokines CCL2 and CXCL8 have been shown to play critical roles for recruitment of inflammatory cells and their expression has been linked to liver fibrosis [37-39]. TGF- $\beta 1$ significantly up regulated CCL2 and
CXCL8 concentrations in cell culture medium, whereas si-FSTL1 inhibited their expression (Figure S4d-e). Importantly, we confirmed that knockdown Fstl1 significantly depressed the expression of $\alpha-S M A$ and Col1 and decreased the cell migration in primary culture of mouse HSCs (Fig. 3h-l). Therefore, these data demonstrated that Fstl1 promotes the activation and transdifferentiation of HSCs in vitro.

\section{Blocking Fstl1 signaling attenuates $\mathrm{CCl}_{4}$ induced liver fibrosis in mice and inhibited TGF- $\beta 1$ activated HSCs in vitro}

Then we examined whether Fstl1 neutralizing antibody (22B6 mAb) would ameliorate $\mathrm{CCl}_{4}$-induced liver fibrosis in vivo, we treated Babl/c mice with $22 \mathrm{~B} 6 \mathrm{mAb}$ or IgG1 along with $\mathrm{CCl}_{4}$ treatment. After 28 days, $22 \mathrm{~B} 6$ $\mathrm{mAb}$ treatment significantly down regulated gene expressions of $\alpha-S M A$, Col1 and Fstl1 (Fig. 4a-c). 22B6 


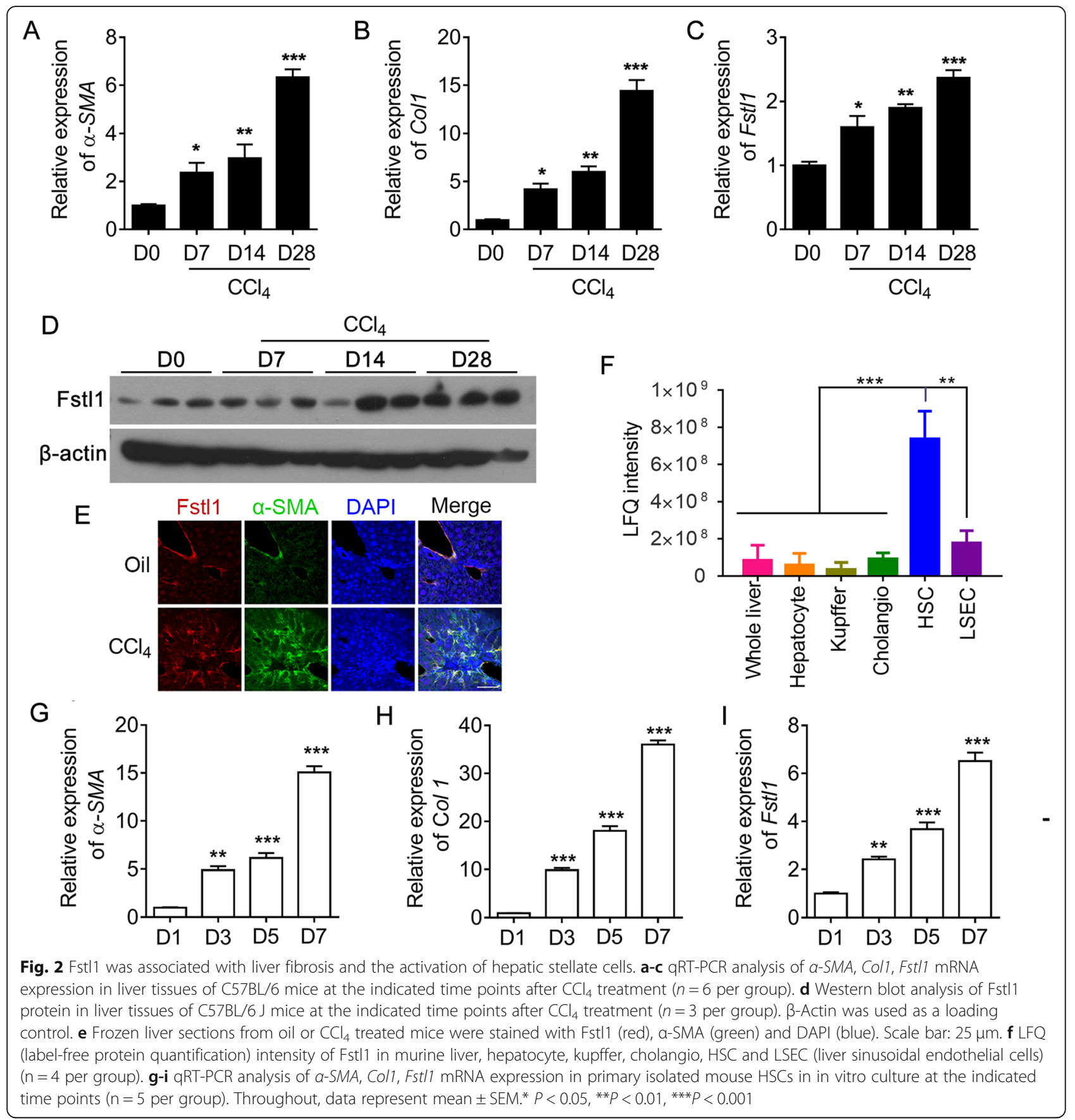

mAb treatment also prevented the development of fibrosis, compared with IgG1 treated mice, as determined by hydroxyproline content, $\alpha$-SMA protein level and collagen staining (Fig. 4d-f). Serum ALT (alanine transaminase) and AST (aspartate aminotransferase) activity were also ameliorated after 22B6 mAb treatment (Fig. 4g-h). Furthermore, 22B6 mAb treatment down regulated phosphorylation of Smad2 and JNK in mouse livers compared with the IgG1 group (Fig. 4f). Thus, we deduced that Fstl1 neutralizing antibody could attenuate
$\mathrm{CCl}_{4}$-induced liver fibrosis in mice through blocking phosphorylation of Smad2/JNK.

We further investigated whether blocking Fstl1 signaling with a neutralizing antibody (22B6 mAb) would inhibit TGF- $\beta 1$ induced activation of HSCs. We found that HSCs activation marker $\alpha$-SMA was down regulated after 22B6 mAb treatment (Figure S5a-b). The activation of TGF- $\beta 1$ signaling measured by phosphorylated Smad2 and JNK were reversed by the 22B6 treatment (Figure $\mathrm{S} 5 \mathrm{a}, \mathrm{c}-\mathrm{d})$. We also found that $22 \mathrm{~B} 6 \mathrm{mAb}$ could 


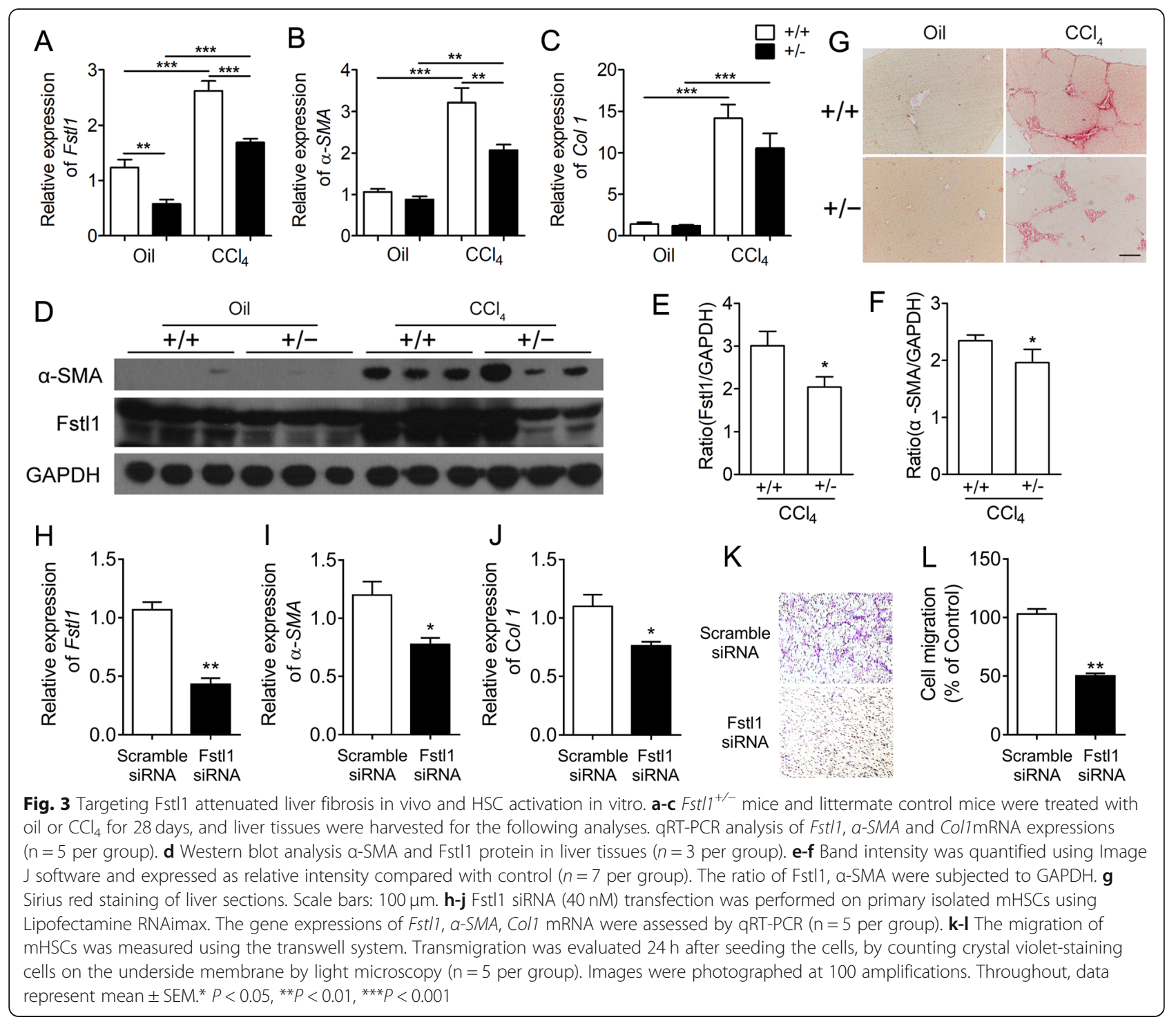

significantly prevent TGF- $\beta 1$ induced ECM production and cell migration compared to isotype matched IgG (IgG1) controls (Fig. S5e-h). Besides, 22B6 mAB significantly decreased chemokine CCL2 and CXCL8 concentrations in cell culture medium compared with IgG (Fig. S5i-j). These data indicate that blocking Fstl1 signaling inhibits TGF- $\beta$ induced HSCs activation, ECM production and cell migration through inhibiting $\mathrm{p}-\mathrm{Smad} 2 / \mathrm{JNK}$.

\section{TGF $\beta 1$-miR29a-Fstl1 regulatory circuit in HSCs}

Consistent with previous studies [16, 17], we found miR29a was down-regulated in response to TGF- $\beta$ stimulation (Figure S2d). Then we explored the relationship among Fstl1, miR29a, and TGF- $\beta 1$ in liver fibrosis. $\mathrm{Fstl1}^{+/-}$mice had significant more expression of miR29a (Fig. 5a). Fstl1 siRNA significantly increased gene expression of miR29a (Fig. 5b), which was independent of
TGF- $\beta 1$ stimulation. Whereas blocking Fstl1 signaling through $22 \mathrm{~B} 6 \mathrm{mAb}$ up regulated miR29a expression in $\mathrm{CCl}_{4}$ treated mice and LX-2 cell line (Fig. $5 \mathrm{c}-\mathrm{d}$ ). Thus, Fstl1 signaling inhibited miR29a expression in HSCs in vitro and in liver fibrosis induced by $\mathrm{CCl}_{4}$ in vivo.

To determine if miR29a direct regulates FSTL1, we used Targetscan to predict consequential pairing of FSTL1 3'UTR target region and subcloned the FSTL1WT and FSTL1-Mut to the luciferase Open reading frame. The result showed that miR29a mimics could suppress luciferase expression when co-transfected with FSTL1-WT plasmid (Figure S6). We also found that miR29a mimics transfection significantly down regulated Fstl1 expression, confirming Fstl1 is a miR29a target. $M i R-29 a$ mimics also significant down-regulated gene expression of Col1, a known direct target of miR29 [16] and $\alpha$-SMA (Fig. 5e,g). In consistent with this, miR29a 


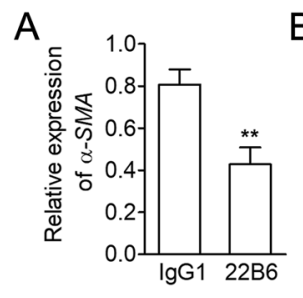

$\mathrm{E}$

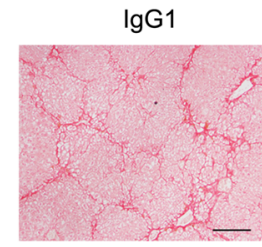

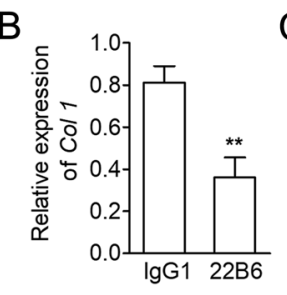

$22 B 6$

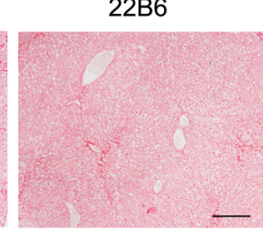

C

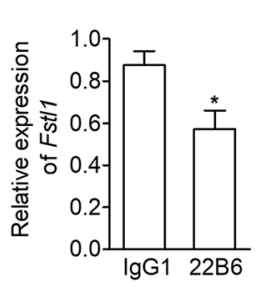

G

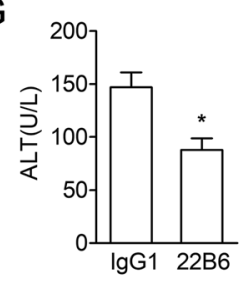

D

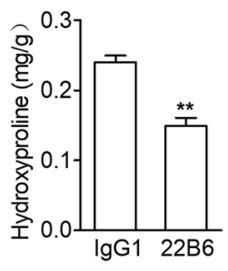

$\mathrm{H}$

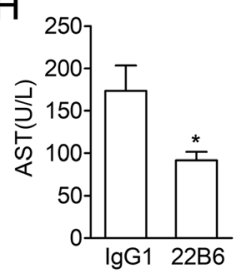

$\mathrm{F}$ $\lg \mathrm{g} 1$ 22B6
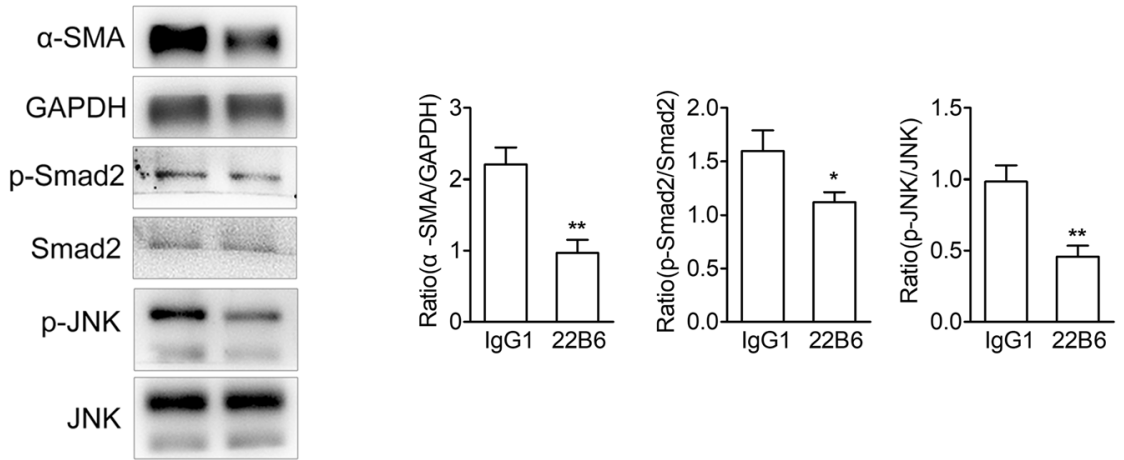

Fig. 4 Fstl1-neutralizing antibody attenuates $\mathrm{CCl}_{4}$-induced liver fibrosis. a-c Babl/c mice were subjected to $\mathrm{CCl}_{4}$ along with $\mathrm{Fstl}_{1}$-neutralizing antibody 22B6 or isotype control lgG1 $(25 \mu \mathrm{g} / \mathrm{mouse}$ ) twice a week, and livers were harvested 4 weeks later for the following analyses ( $n=6 \mathrm{mice}$ per group). qRT-PCR analysis of $a-S M A$, Col1 and Fst/1 mRNA expressions. d Hydroxyproline contents in liver tissues were measured. e Sirius red staining of liver sections. Scale bars, $100 \mu \mathrm{m}$. f Protein expression levels of a-SMA, p-Smad2, Smad2, p-JNK, JNK in liver tissues were assessed by Western blot. Band intensity was quantified using Image J software and expressed as relative intensity compared with control. The ratio of a-SMA were subjected to GAPDH. The ratio of p-Smad2 was subjected to Smad2. The ratio of p-JNK was subjected to JNK. (G,H) Serum levels of ALT and AST were measured. Throughout, data represent means \pm SEM. ${ }^{*} P<0.05,{ }^{* *} P<0.01$

inhibitor up-regulated expression of Fstl1, $\alpha$-SMA and Col1 (Fig. 5f,h).

To test whether TGF- $\beta 1$ induces Fstl1 through down regulation of miR29a, we used miR29a mimics or inhibitor in TGF- $\beta 1$ stimulated human LX- 2 cells. TGF- $\beta 1$ induced Fstl1, $\alpha$-SMA and Col1 expression, whereas decreased miR29a expression (Fig. 5d-h). MiR-29a mimics partially blocked, while miR29a inhibitor further enhanced TGF- $\beta 1$ induced Fstl1 expression (Fig. $5 d-h$ ). All together, these data suggest that TGF- $\beta 1$ might induce Fstl1 partially through down regulation of miR29a, while Fstl1 and miR29a reciprocally regulate each other in HSCs.

\section{Discussion}

This work highlights the importance of Fstl1 in liver fibrosis. Fstl1 was up regulated in human and mouse fibrotic livers. In the genetic models used, we demonstrated that Fstl1-haplodeficiency mice were less susceptible to chemically induced liver fibrosis. Knockdown Fstl1 significantly inhibited TGF- $\beta 1$ stimulated cell migration and ECM accumulation. Fstl1-neutraliztion antibody had anti-fibrotic effect in vivo and inhibited HSCs activation and migration in vitro through inhibiting $\mathrm{p}$-Smad2/JNK. Furthermore, we demonstrated that there was a TGF- $\beta 1-$ miR29a-Fstl1 regulatory circuit mediating liver fibrosis. These results are in agreement with previous studies showing Fstl1 was induced in liver fibrosis by $\mathrm{CCl}_{4}$ treatment [40]. Our observation also confirmed the previous studies that knockdown Fstl1 attenuate liver fibrosis [25, 26].

TGF- $\beta$ signaling plays a critical role in the regulation of cell growth, migration and differentiation and is a central driver in liver fibrosis [1]. Classical TGF- $\beta$ signaling is initiated with ligand-induced oligomerization of serine/threonine receptor kinases and phosphorylation 

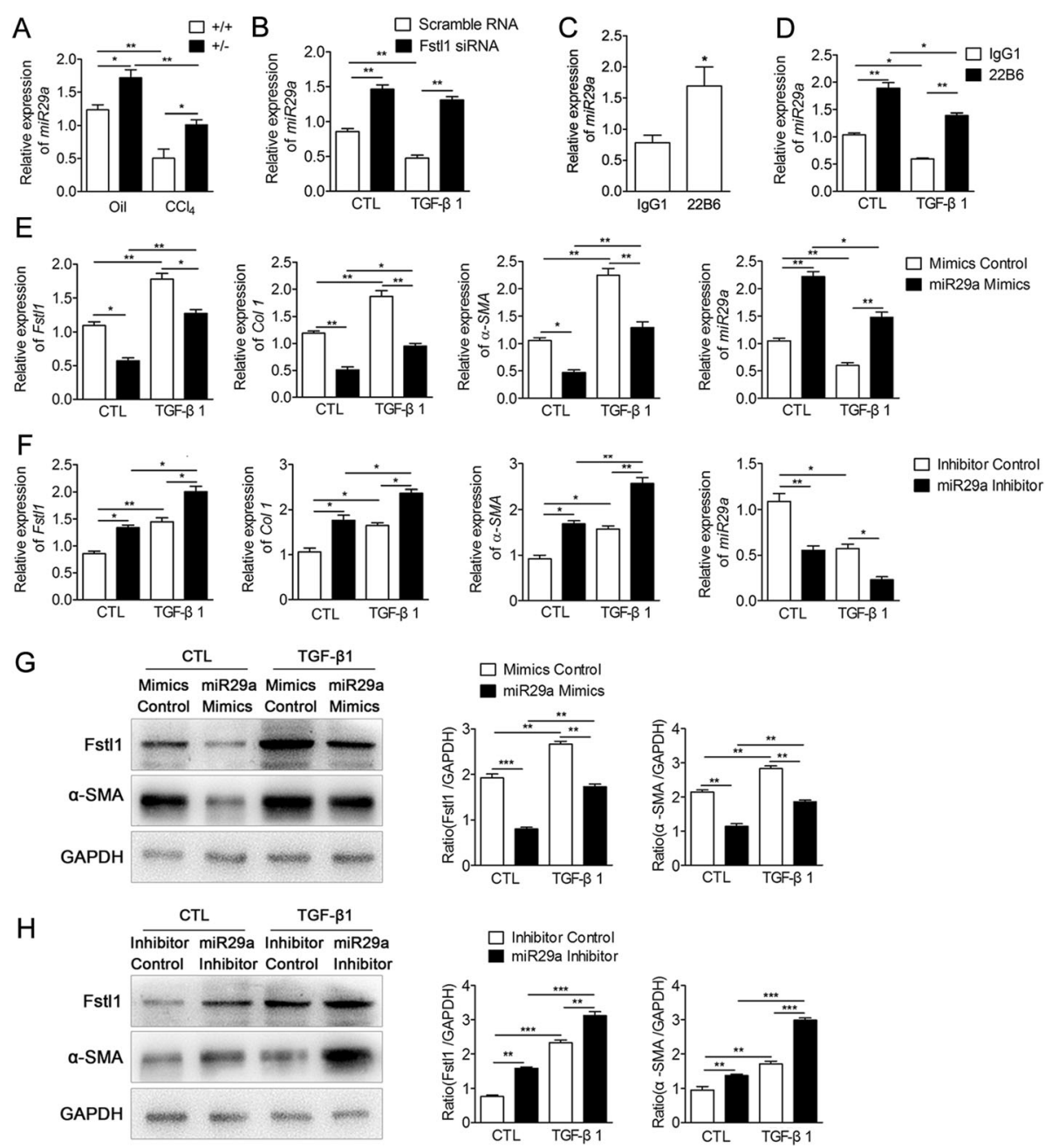

Fig. 5 TGF- $\beta 1$-miR29a-Fstl1 Regulatory Circuit Mediates Fibrosis. a The livers of $\mathrm{Fs}_{\mathrm{s}} / 1^{+/-}$mice were harvested for the analyses. The expression of miR29a was assessed by qRT-PCR ( $n=3$ per group). b Fst11 siRNA ( $40 \mathrm{nM}$ ) transfection was performed on LX-2 cells using Lipofectamine RNAimax ( $n=3$ per group). The expression of miR29a was assessed by qRT-PCR ( $n=3$ per group). c BABL/c mice were subjected to $\mathrm{CCl}_{4}$ along with $\mathrm{Fstl}_{1}$ neutralizing antibody $22 \mathrm{~B} 6$ or isotype control $\operatorname{lgG} 1(25 \mu \mathrm{g} / \mathrm{mouse})$ twice a week, and livers were harvested 4 weeks later for the analyses. The expression of miR29a was assessed by qRT-PCR ( $n=6$ per group). $\mathbf{d} L X-2$ cells were treated with $22 B 6$ or isotype control lgG1 $(2 \mu \mathrm{g} / \mathrm{ml})$. The expression of miR29a was assessed by qRT-PCR ( $n=3$ per group). e MiR29a mimics ( $100 \mathrm{nM}$ ) were transiently transfected into LX-2 cells for $48 \mathrm{~h}$. The expression of Fst11, Col1, a-SMA mRNA and miR29a were assessed by qRT-PCR ( $\mathrm{n}=3$ per group). $\mathbf{f}$ MiR29a inhibitors (200 nM) were transiently transfected into LX-2 cells for $48 \mathrm{~h}$. The expression of Fstll, Coll, a-SMA mRNA and miR29a were assessed by qRT-PCR ( $\mathrm{n}=3$ per group). g-h Protein expression levels of a-SMA and Fstl1 in cell extracts were assessed by Western blot. Band intensity was quantified using Image J software and expressed as relative intensity compared with control. The ratio of Fstl1 and a-SMA were subjected to GAPDH ( $n=2$ per group). Throughout, data represent means \pm SEM. ${ }^{*} P<0.05,{ }^{* *} P<0.01,{ }^{* * *} P<0.001$

of downstream cytoplasmic signaling molecules Smad2 and Smad3 [41, 42]. TGF- $\beta$ signaling can also affect Smad-independent pathways, such as the MAPK and Akt signaling pathways [4, 41, 43, 44]. Fstl1 was shown as a pro-migratory factor enhancing ERK phosphorylation and regulated by miR-198 in wound healing [45]. Our results implicate that blocking Fstl1 by neutralizing antibody in mice with $\mathrm{CCl}_{4}$ treatment also modulates the Smad2 and JNK signaling pathways.

Inflammation is typically present in all chronic liver diseases and associated with the development of fibrosis
[46]. FSTL1 has been identified as a novel proinflammatory protein and systemic administration of adenoviral vectors expressing Fstl1 (Ad-Fstl1) to mice induced expression of proinflammatory cytokines in liver and exacerbated collagen-induced arthritis [47]. Conversely, adenovirus-mediated administration of Fstl1 to WT mice with subtotal nephrectomy ameliorated tubulointerstitial fibrosis and reduced expression of proinflammatory mediators in the remnant kidney [22]. 4methylumbelliferone, an inhibitor of hyaluronan deposition, suppressed the HSC trans-differentiation and 
altered macrophage localization with the downregulation of Fstl1 in $\mathrm{CCl}_{4}$ treated mice [40]. Further studies are needed to determine whether Fstl1 modulates inflammatory responses in liver fibrosis.

Overexpressing miR-29a/b markedly reduced the degree of liver fibrosis induced by $\mathrm{CCl}_{4}$ in mice and decreased collagen expression in LX-2 cells [10, 48]. Ectopic expression of miR-29b in activated HSCs also blunted the increased expression of $\alpha$-SMA, caused cell cycle arrest, and induced apoptosis through targeting PI3K/AKT pathway [11]. Furthermore, Knockout miR29 enhanced mortality and the expression of fibrotic markers in mouse livers with $\mathrm{CCl}_{4}$ treatment [49]. Consistent with this, our studies found miR29a mimics significantly decreased COL1, $\alpha$-SMA and FSTL1 expression, while miR29a inhibitors showed the reverse effects. On the other hand, knockdown Fstl1 in LX-2 significantly increased expression of miR29a. We also proved that miR29a directly targeted FSTL1 3'UTR. Due to sequence similarity, $m i R 29 b$ and $m i R 29 c$ might also target Fstl1 3'UTR. Blocking Fstl1 signaling through $22 \mathrm{~B} 6 \mathrm{mAb}$ up regulated $m i R 29 a$ expression in $\mathrm{CCl}_{4}$ treated mice. These data suggest that Fstll modulates Smad/JNK phosphorylation and miR29a in HSC in vitro and in $\mathrm{CCl}_{4}$ induced liver fibrosis in mice. There is also a cross talk between Fstl1 and miR29a signaling.

We acknowledge several limitations. FSTL1 is a secreted protein which may interact with various extracellular molecules or transmembrane receptors. FSTL1 stimulated the survival and migration of endothelial cells through the cell surface receptor of DIP2A [50]. FSTL1 directly interacted with the secreted phosphoprotein 1 (SPP1)/osteopontin and led to inactivation of integrin/ CD44-associated signaling [51]. So Fstl1 may also act on endothelial cells or other cell types in liver fibrosis. Moreover, the mechanisms by which Fstl1 modulates miR29a expression remain unresolved.

\section{Conclusion}

Here, we provide evidence that targeting Fstl1 inhibits the activation of $\mathrm{HSCs}$ and ameliorates $\mathrm{CCl}_{4}$-induced liver fibrosis in mice by modulating TGF- $\beta 1-m i R 29 a-$ Fstl1 regulatory circuit and downstream Smad2/JNK signaling in activated HSCs. Fstl1 may serve as a novel therapeutic approach in the treatment for patients with severe liver fibrosis.

\section{Supplementary information}

Supplementary information accompanies this paper at https://doi.org/10. 1186/s12964-020-00610-0.

Additional file 1 : Table S1. Characteristics and serum Follistatin-like protein 1 (FSTL1) levels of subjects investigated. Figure S1. FSTL1 Expression in Human Activated HSCS and Senescent HSCs. Figure S2. TGF- $\beta 1$
Induced Fstl1 gene expression in a time-and dose-dependent manner and downregulated miR29a in human LX-2 cell Line. Figure S3. TGF-B1 Induced Fstl 1 Gene Expression in a time-and dose-dependent manner in rat CFSC-8B cell line. Figure S4. Knockdown of Fstl1 attenuated the activation of $L X-2$ cells. Figure S5. Fstl1-neutralizing antibody reduced $L X-2$ cell migration, chemokine secretion and inhibiting TGF- $31 / \mathrm{Smad} 2 / \mathrm{JNK}$ Signaling. Figure S6. MiR29a targets Fstl1 3'UTR.

\section{Abbreviations}

HSCs: Hepatic stellate cells; Fst11: Follistatin like 1; TGF- $\beta 1$ : Transforming growth factor- 1 1; ECM: Extracellular matrix; miR29a: microRNA-29a; NAFL D: Nonalcoholic fatty liver disease; Fst: Follistatin; SPARC: Secreted protein acidic rich in cysteines; $\mathrm{CCl}_{4}$ : Carbon tetrachloride; a-SMA: a-smooth muscle actin; Col1: Collagen I; CCL2: C-C Motif Chemokine Ligand 2; CXCL8: C-X-C Motif Chemokine Ligand 8; PSR: Picro-sirius red; qRT-PCR: Real-time quantitative polymerase chain reaction; ALT: Alanine aminotransferase; AST: Aspartate aminotransferase; ANOVA: one-way analysis of variance; CTL: Control; HBV: Viral hepatitis B; LC: Cirrhosis; HCC: Hepatocellular carcinoma; LSECs: Liver sinusoidal endothelial cells

\section{Acknowledgments}

We thank Prof. Wen Ning (from Nankai University) for providing the Fst/1 knockout lines, the FSTL1 neutralizing antibody 22B6 and for critical reading the manuscript.

\section{Authors' contributions}

All authors have contributed substantially to this work. YG, YD, XL, FJ, YR, YD, and LL conducted the experiments; $\mathrm{H}-\mathrm{YX}, \mathrm{Z}-\mathrm{ML}$, and J-SS analyzed the data. $Y R$, and $X X$ collected blood samples, $Y G, L L$ and Z-HX designed the experiments. YG, YD, and DJ wrote the paper. All authors read and approved the final version of this manuscript.

\section{Funding}

This work was supported by National Natural Science Foundation of China (NSFC) grants 31771514, 31970746, 31201020 (to YG), National first-class discipline program of Light Industry Technology and Engineering (LITE2018-11), and Fundamental Research Funds for the Central Universities (Grant No. JUSRP22029, JUSRP21824, JUSRP 22047).

\section{Availability of data and materials}

All data generated or analyzed during this study are included either in this article or in the supplementary Materials and Methods, Tables, Figures and Figure Legends files.

\section{Ethics approval and consent to participate}

The human study was approved by the Institutional Review Board of Wuxi No.2 People's Hospital (No. 20170608) and were in accordance with the principles of the Declaration of Helsinki as revised in 2000. All human tissues are collected under IRB and HIPPA approved protocols, and approved for commercial product development. All animal experiments were reviewed and authorized by the Animal Research Committee of Jiangnan University and Nankai University.

Consent for publication

Not applicable.

\section{Competing interests}

The authors have declared that no competing interest exists.

\section{Author details}

${ }^{1}$ School of Pharmaceutical Sciences, Jiangnan University, Wuxi 214122, China. ${ }^{2}$ National Engineering Laboratory for Cereal Fermentation Technology, Jiangnan University, Wuxi, Jiangsu, China. Jiangsu Engineering Research Center for Bioactive Products Processing Technology, Jiangnan University, Wuxi 214122, P.R. China. ${ }^{4}$ State Key Laboratory of Medicinal Chemical Biology, College of Life Sciences, Nankai University, Tianjin 300071, China. ${ }^{5}$ Department of Medicine, Cedars-Sinai Medical Center, Los Angeles, CA 90048, USA. ${ }^{6}$ Cambridge-Suda Genomic Resource Center, Soochow University, Suzhou 215123, China. 'Wuxi No. 2 People's Hospital, Wuxi 214002, China. 


\section{Received: 21 February 2020 Accepted: 8 June 2020}

\section{Published online: 15 September 2020}

\section{References}

1. Bataller R, Brenner DA. Liver fibrosis. J Clin Investig. 2005;115(2):209-18

2. Tsuchida T, Friedman SL. Mechanisms of hepatic stellate cell activation. Nat Rev Gastroenterol Hepatol. 2017;14(7):397-411.

3. Czaja AJ. Hepatic inflammation and progressive liver fibrosis in chronic liver disease. World J Gastroenterol. 2014;20(10):2515-32.

4. Gressner AM, Weiskirchen R, Breitkopf K, Dooley S. Roles of TGF-beta in hepatic fibrosis. Front Biosci. 2002;7:d793-807.

5. De Minicis S, Seki E, Uchinami H, Kluwe J, Zhang Y, Brenner DA, et al. Gene expression profiles during hepatic stellate cell activation in culture and in vivo. Gastroenterology. 2007;132(5):1937-46.

6. Seki E, De Minicis S, Osterreicher CH, Kluwe J, Osawa Y, Brenner DA, et al. TLR4 enhances TGF-beta signaling and hepatic fibrosis. Nat Med. 2007; 13(11):1324-32.

7. Kanzler S, Lohse AW, Keil A, Henninger J, Dienes HP, Schirmacher P, et al. TGF-beta1 in liver fibrosis: an inducible transgenic mouse model to study liver fibrogenesis. Am J Phys. 1999;276(4 Pt 1):G1059-68.

8. Liu X, Hu H, Yin JQ. Therapeutic strategies against TGF-beta signaling pathway in hepatic fibrosis. Liver Int. 2006;26(1):8-22

9. Kriegel AJ, Liu Y, Fang Y, Ding X, Liang M. The miR-29 family: genomics, cell biology, and relevance to renal and cardiovascular injury. Physiol Genomics. 2012:44(4):237-44.

10. Roderburg C, Urban GW, Bettermann K, Vucur M, Zimmermann H, Schmidt $\mathrm{S}$, et al. Micro-RNA profiling reveals a role for miR-29 in human and murine liver fibrosis. Hepatology. 2011;53(1):209-18.

11. Wang J, Chu ES, Chen HY, Man K, Go MY, Huang XR, et al. microRNA-29b prevents liver fibrosis by attenuating hepatic stellate cell activation and inducing apoptosis through targeting PI3K/AKT pathway. Oncotarget. 2015; 6(9):7325-38.

12. Cushing L, Kuang PP, Qian J, Shao FZ, Wu JJ, Little F, et al. miR-29 is a major regulator of genes associated with pulmonary fibrosis. Am J Resp Cell Mol. 2011:45(2):287-94.

13. Xie T, Liang J, Geng Y, Liu N, Kurkciyan A, Kulur V, et al. MicroRNA-29c prevents pulmonary fibrosis by regulating epithelial cell renewal and apoptosis. Am J Respir Cell Mol Biol. 2017.

14. van Rooij E, Sutherland LB, Thatcher JE, DiMaio JM, Naseem RH, Marshall WS, et al. Dysregulation of microRNAs after myocardial infarction reveals a role of miR-29 in cardiac fibrosis. P Natl Acad Sci USA. 2008;105(35):1302732.

15. Zhang Y, Wu L, Wang Y, Zhang M, Li L, Zhu D, et al. Protective role of estrogen-induced miRNA-29 expression in carbon tetrachloride-induced mouse liver injury. J Biol Chem. 2012;287(18):14851-62.

16. Hambrock HO, Kaufmann B, Muller S, Hanisch FG, Nose K, Paulsson M, et al. Structural characterization of TSC-36/Flik: analysis of two charge isoforms. J Biol Chem. 2004;279(12):11727-35.

17. Shibanuma M, Mashimo J, Mita A, Kuroki T, Nose K. Cloning from a mouse osteoblastic cell line of a set of transforming-growth-factor-beta 1-regulated genes, one of which seems to encode a follistatin-related polypeptide. Eur J Biochem. 1993;217(1):13-9.

18. Patella S, Phillips DJ, Tchongue J, de Kretser DM, Sievert W. Follistatin attenuates early liver fibrosis: effects on hepatic stellate cell activation and hepatocyte apoptosis. Am J Physiol Gastrointest Liver Physiol. 2006;290(1): G137-44.

19. Camino AM, Atorrasagasti C, Maccio D, Prada F, Salvatierra E, Rizzo M, et al Adenovirus-mediated inhibition of SPARC attenuates liver fibrosis in rats. J Gene Med. 2008;10(9):993-1004.

20. Geng Y, Dong Y, Yu M, Zhang L, Yan X, Sun J, et al. Follistatin-like 1 (Fstl1) is a bone morphogenetic protein (BMP) 4 signaling antagonist in controlling mouse lung development. Proc Natl Acad Sci U S A. 2011;108(17):7058-63.

21. Dong YY, Geng Y, Li L, Li XH, Yan XH, Fang YS, et al. Blocking follistatin-like 1 attenuates bleomycin-induced pulmonary fibrosis in mice. J Exp Med. 2015;212(2):235-52.

22. Hayakawa S, Ohashi K, Shibata R, Kataoka Y, Miyabe M, Enomoto T, et al. Cardiac myocyte-derived follistatin-like 1 prevents renal injury in a subtotal nephrectomy model. J Am Soc Nephrol. 2015;26(3):636-46.

23. Wei K, Serpooshan V, Hurtado C, Diez-Cunado M, Zhao M, Maruyama S, et al. Epicardial FSTL1 reconstitution regenerates the adult mammalian heart. Nature. 2015;525(7570):479-85.
24. Wu Y, Zhou S, Smas CM. Downregulated expression of the secreted glycoprotein follistatin-like 1 (Fstl1) is a robust hallmark of preadipocyte to adipocyte conversion. Mech Dev. 2010;127(3-4):183-202.

25. Vollmann EH, Cao L, Amatucci A, Reynolds T, Hamann S, Dalkilic-Liddle I, et al. Identification of novel fibrosis modifiers by in vivo siRNA silencing. Mol Ther Nucleic Acids. 2017;7:314-23.

26. Shang H, Liu X, Guo H. Knockdown of Fstl1 attenuates hepatic stellate cell activation through the TGFbeta1/Smad3 signaling pathway. Mol Med Rep. 2017;16(5):7119-23.

27. Galimov A, Hartung A, Trepp R, Mader A, Fluck M, Linke A, et al. Growth hormone replacement therapy regulates microRNA-29a and targets involved in insulin resistance. J Mol Med (Berl). 2015;93(12):1369-79.

28. Ma R, Wang M, Gao S, Zhu L, Yu L, Hu D, et al. miR-29a Promotes the Neurite Outgrowth of Rat Neural Stem Cells by Targeting Extracellular Matrix to Repair Brain Injury. Stem Cells Dev. 2020;29(9):599-614.

29. Iredale JP. Models of liver fibrosis: exploring the dynamic nature of inflammation and repair in a solid organ. J Clin Invest. 2007;117(3):539-48.

30. Jiang D, Liang J, Hodge J, Lu B, Zhu Z, Yu S, et al. Regulation of pulmonary fibrosis by chemokine receptor CXCR3. J Clin Invest. 2004;114(2):291-9.

31. Mederacke I, Dapito DH, Affo S, Uchinami H, Schwabe RF. High-yield and high-purity isolation of hepatic stellate cells from normal and fibrotic mouse livers. Nat Protoc. 2015;10(2):305-15.

32. Yang $W$, Wu Y, Wang $C$, Liu Z, Xu M, Zheng X. FSTL1 contributes to tumor progression via attenuating apoptosis in a AKT/GSK-3beta - dependent manner in hepatocellular carcinoma. Cancer Biomark. 2017;20(1):75-85.

33. Moylan CA, Pang H, Dellinger A, Suzuki A, Garrett ME, Guy CD, et al. Hepatic gene expression profiles differentiate presymptomatic patients with mild versus severe nonalcoholic fatty liver disease. Hepatology. 2014;59(2):471-82.

34. Azimifar SB, Nagaraj N, Cox J, Mann M. Cell-type-resolved quantitative proteomics of murine liver. Cell Metab. 2014;20(6):1076-87.

35. Xu L, Hui AY, Albanis E, Arthur MJ, O'Byrne SM, Blaner WS, et al. Human hepatic stellate cell lines, LX-1 and LX-2: new tools for analysis of hepatic fibrosis. Gut. 2005;54(1):142-51.

36. Krizhanovsky V, Yon M, Dickins RA, Hearn S, Simon J, Miething C, et al. Senescence of activated stellate cells limits liver fibrosis. Cell. 2008;134(4): 657-67.

37. Seki E, De Minicis S, Gwak GY, Kluwe J, Inokuchi S, Bursill CA, et al. CCR1 and CCR5 promote hepatic fibrosis in mice. J Clin Invest. 2009;119(7):1858-70.

38. Holt AP, Haughton EL, Lalor PF, Filer A, Buckley CD, Adams DH. Liver myofibroblasts regulate infiltration and positioning of lymphocytes in human liver. Gastroenterology. 2009;136(2):705-14.

39. Zimmermann HW, Seidler S, Gassler N, Nattermann J, Luedde T, Trautwein C, et al. Interleukin-8 is activated in patients with chronic liver diseases and associated with hepatic macrophage accumulation in human liver fibrosis. PLoS One. 2011;6(6):e21381.

40. Andreichenko IN, Tsitrina AA, Fokin AV, Gabdulkhakova Al, Maltsev DI, Perelman GS, et al. 4-methylumbelliferone Prevents Liver Fibrosis by Affecting Hyaluronan Deposition, FSTL1 Expression and Cell Localization. Int J Mol Sci. 2019;20(24):6301.

41. Lechuga CG, Hernandez-Nazara ZH, Dominguez Rosales JA, Morris ER, Rincon AR, Rivas-Estilla AM, et al. TGF-beta1 modulates matrix metalloproteinase-13 expression in hepatic stellate cells by complex mechanisms involving p38MAPK, PI3-kinase, AKT, and p70S6k. Am J Physiol Gastrointest Liver Physiol. 2004;287(5):G974-87.

42. Meng XM, Tang PM, Li J, Lan HY. TGF-beta/Smad signaling in renal fibrosis. Front Physiol. 2015;6:82.

43. Koren J 3rd, Jinwal UK, Jin Y, O'Leary J, Jones JR, Johnson AG, et al. Facilitating Akt clearance via manipulation of Hsp70 activity and levels. J Biol Chem. 2010;285(4):2498-505.

44. Wang Y. Phosphatidylinositol 3-kinase/Akt pathway regulates hepatic stellate cell apoptosis. World J Gastroenterol. 2008;14(33):5186.

45. Sundaram GM, Ismail HM, Bashir M, Muhuri M, Vaz C, Nama S, et al. EGF hijacks miR-198/FSTL1 wound-healing switch and steers a two-pronged pathway toward metastasis. J Exp Med. 2017.

46. Seki E, Schwabe RF. Hepatic inflammation and fibrosis: functional links and key pathways. Hepatology. 2015;61(3):1066-79.

47. Miyamae T, Marinov AD, Sowders D, Wilson DC, Devlin J, Boudreau R, et al. Follistatin-like protein-1 is a novel proinflammatory molecule. J Immunol. 2006;177(7):4758-62

48. Bian EB, Li J, Zhao B. miR-29, a potential therapeutic target for liver fibrosis. Gene. 2014;544(2):259-60. 
49. Kogure T, Costinean S, Yan I, Braconi C, Croce C, Patel T. Hepatic miR-29ab1 expression modulates chronic hepatic injury. J Cell Mol Med. 2012;16(11): 2647-54.

50. Ouchi N, Asaumi Y, Ohashi K, Higuchi A, Sono-Romanelli S, Oshima Y, et al. DIP2A functions as a FSTL1 receptor. J Biol Chem. 2010;285(10):7127-34.

51. Chiou J, Chang YC, Tsai HF, Lin YF, Huang MS, Yang CJ, et al. Follistatin-like protein 1 inhibits lung Cancer metastasis by preventing Proteolytic activation of Osteopontin. Cancer Res. 2019;79(24):6113-25.

\section{Publisher's Note}

Springer Nature remains neutral with regard to jurisdictional claims in published maps and institutional affiliations.

Ready to submit your research? Choose BMC and benefit from:

- fast, convenient online submission

- thorough peer review by experienced researchers in your field

- rapid publication on acceptance

- support for research data, including large and complex data types

- gold Open Access which fosters wider collaboration and increased citations

- maximum visibility for your research: over $100 \mathrm{M}$ website views per year

At $\mathrm{BMC}$, research is always in progress.

Learn more biomedcentral.com/submissions 